\title{
Parametric Robot Search System for Mines Based on the Interaction of Electromagnetic and Seismic Waves
}

\author{
Roman G. Shaydurov* \\ Siberian Federal University \\ 79 Svobodny, Krasnoyarsk, 660041, Russia
}

Received 19.04.2018, received in revised form 14.09.2018, accepted 12.10.2018

\begin{abstract}
A brief review of remote parametric methods for searching mines and minefields. The physical principles of a remote method for detecting subsurface objects of the mines type in the ground and in the aqueous medium are described on the basis of excitation along the interface between the Rayleigh shock waves and the registration of the Doppler phase shift of the radar signal that occurs when the body of the object is vibrated under the action of seismic impacts. The reasons for the hardware implementation of the new method are outlined, the quantitative correlations in the radio channel of the radar system are estimated.
\end{abstract}

Keywords: seismic wave, vibrational source, parametric method, radar.

Citation: Shaydurov R.G. Parametric robot search system for mines based on the interaction of electromagnetic and seismic waves, J. Sib. Fed. Univ. Eng. technol., 2018, 11(7), 755-763. DOI: 10.17516/1999-494X-0089.

\section{Параметрическая роботизированная}

\section{система поиска мин на основе взаимодействия}

электромагнитных и сейсмических волн

\section{Р.Г. Шайдуров}

Сибирский федеральный университет

Россия, 660041, Красноярск, пр. Свободныгй, 79

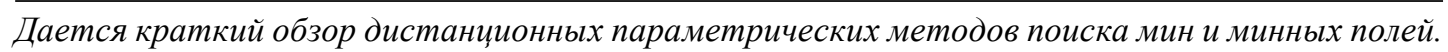
Описываются физические основы дистаниионного метода обнаружения подповерхностных объектов типа мин в земле на основе возбуждения вдоль границы раздела земля-воздух ударных сейсмических волн Релея и регистрации радиолокационным методом доплеровского сдвига фаз, возникающего под действием сейсмических ударов вибраций корпуса объекта

(C) Siberian Federal University. All rights reserved

This work is licensed under a Creative Commons Attribution-NonCommercial 4.0 International License (CC BY-NC 4.0).

* Corresponding author E-mail address: rshaydurov@sfu-kras.ru 
поиска. Изложены соображения по аппаратной реализации нового метода, дается оценка количественных соотношений в радиоканале радиолокационной системы.

Ключевые слова: сейсмическая волна, вибрационный источник, параметрический метод, радар.

\section{Введение}

Проблема увеличения производительности работ при гуманитарном разминировании больших площадей особенно остро встала в последнее время в связи с локальными войнами на Кавказе, Средней Азии, Ближнем Востоке и др. При этом обеспечение безопасности саперов является одним из главных критериев эффективности работ.

По опыту разминирования в Сирии можно утверждать, что основными средствами обнаружения мин и боеприпасов являются индивидуальные миноискатели, переносимые саперами. В связи с недостаточной условной вероятностью их правильного обнаружения, при высокой условной вероятности «ложных тревог», скорость передвижения саперов не превышает 0,1-0,5 м/с. Это, конечно, во многом зависит от тактико-технических параметров используемых миноискателей. На сегодня самыми распространенными переносимыми миноискателями признаны радиоволновые, основанные на измерениях электропроводности и диэлектрической проницаемости почвы в широком диапазоне частот в пределах 300-3000 МГц [1]. В печати сообщалось также о разработке голографических подповерхностных радиолокаторов (МГТУ им. Баумана) и видеоимпульсных, работающих с наносекундными импульсами зондирования.

В $[2,3]$ сообщалось о работах по созданию параметрических миноискателей, основанных на возбуждении корпусов мин сейсмическими волнами и считывании вибрации поверхности почвы с помощью радиолокатора [3], либо лазера [4].

Этот метод позволяет получить дополнительный признак обнаружения мин с использованием собственных частот механических колебаний их корпусов, что в комплексе с радиоволновым методом улучшает тактико-технические параметры поиска.

Целью статьи является рассмотрение возможностей построения роботизированного комплекса разминирования больших площадей, обеспечивающего максимальную безопасность работ саперов с одновременным увеличением производительности. За счет дублирования классических радиоволновых миноискателей параметрической радиолокационной системой повышается вероятность распознавания целей на фоне различных мешающих предметов.

\section{Исходные предпосылки}

Возможный вариант подобного комплекса, включающий базовую машину с командой операторов и несколько управляемых на расстоянии подвижных блоков-роботов (БР) с навесными миноискателями различных классов, изображен на рис. 1.

На базовой машине кроме команды операторов, управляющих индивидуально каждым из БР, размещены передатчик радиолокатора с дальностью действия 100-200 м и навесной сейсмический вибратор с рабочим диапазоном частот в пределах механических резонансов корпусов поисковых объектов - мин и других взрывных устройств, в частности фугасов, авиабомб и пр.

Поисковыми элементами на борту БР являются набор приемников с антеннами для приема отраженных от почвы радиолокационных сигналов, излучаемых передатчиком РЛС с базовой 
машины, и радиоволновые датчики. Система синхронизации обеспечивает временное разделение сигналов комплекса, при котором вначале используется параметрическая система с передатчиком РЛС и затем радиоволновые датчики. Примерная конструкция блока-робота приведена на рис. 2.

Все данные наблюдений по обоим каналам передаются на компьютер оператора по проводной связи либо по радиотракту.

При обнаружении мины оператор останавливает БР, отводит на безопасное расстояние и расстреливает объект поиска из бортового пулемета или другого средства уничтожения.

Такова в общем виде конструкция комплекса и его алгоритм работы.

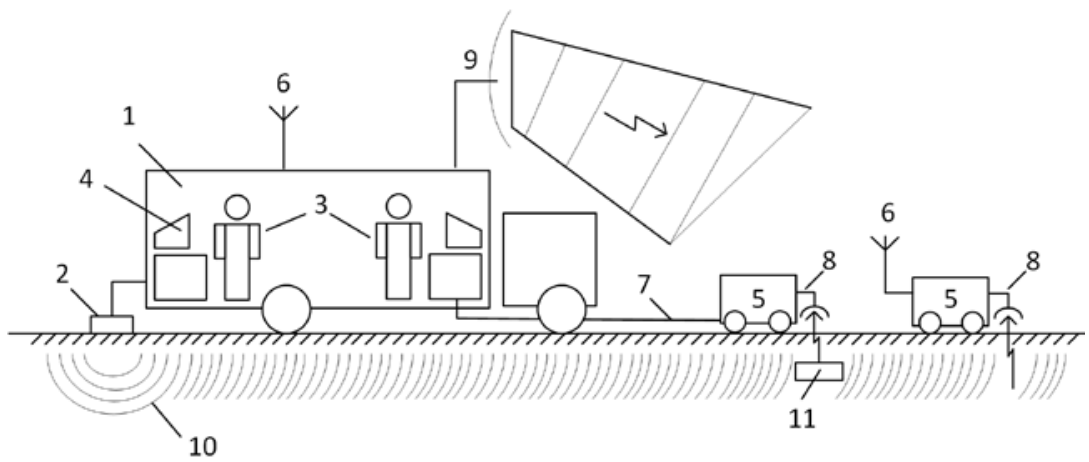

Рис. 1. Возможный вариант робототехнического комплекса: 1 - базовая машина; 2 - сейсмический вибратор; 3 - операторы БР; 4 - пульт управления БР; 5 - БР; 6 - радиоканал управления БР; 7 - проводная линия управления БР; 8 - совмещенный миноискатель; 9 - антенна бортовой РЛС; 10 - сейсмическая волна; 11 - объект поиска

Fig. 1. Possible version of the robotic complex: 1 - basic machine; 2 - seismic vibrator; 3 - RU operators; 4 - RU remote control; 5 - the robot unit RU; 6 - RU control radio channel; 7 - wire control line RU; 8 - combined mine detector; 9 - onboard radar antenna; 10 - seismic wave; 11 - search object

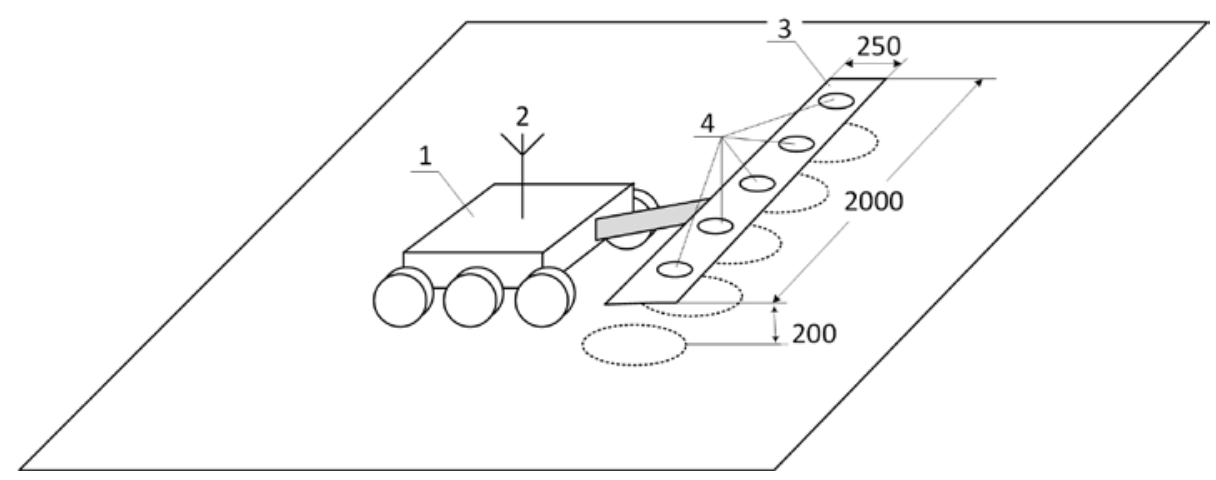

Рис. 2. Конструкция блока-робота: 1 - корпус БР; 2 - антенна управляющего радиоканала; 3 - рама; 4 - антенны параметрического и радиоволнового миноискателей

Fig. 2. The design of the the robot unit: 1 - carcass RU; 2 - control radio channel antenna; 3 - frame; 4 - antenna of parametric and radio wave mine detectors 


\section{Оценка тактико-технических характеристик параметрического канала}

На рис. 3 изображена схема одновременного возбуждения полупространства земля-воздух сейсмическими волнами Релея и электромагнитными волнами со считыванием отраженных сигналов в точке облучения.

Под действием сейсмического (акустического) вибратора, состоящего из ударно-волнового датчика - 1, возбуждаемого генератором - 2, в поверхностном слое земли создается сейсмическая волна Релея - 3, распространяющаяся вдоль со скоростью $v_{a}$. Через некоторое время $t_{a}=\frac{r}{v_{a}}$, где $r$ - расстояние между точкой излучения и объектом поиска (мины) -4, с направленной антенны -5 радиопередатчика -6 излучается квазинепрерывный радиосигнал -7 в сторону объекта, а отраженный электромагнитный сигнал от поверхности почвы принимается антенными элементами БР -8 и после обработки входным каскадом приемника -9 передается по каналу связи - 10, 11, 12 на приемоиндикатор оператора - 13.

Временная диаграмма излучения и приема сигналов представлена на рис. 4.

Импульсы излучения сейсмического вибратора - 1 повторяются с периодом $T_{a}$, устанавливаемым заранее в зависимости от плотности грунта и дальности поиска. Через время задержки $t_{P}$, после окончания работы вибратора на отрезок времени $\Delta t_{P}$ включается передатчик радиоволнового миноискателя - 2, входящего в комплекс. Это позволяет исключить влияние на радиоволновый канал вибрации грунта. В момент - 3 включается приемник РЛС параметрического миноискателя, обрабатывающего отраженные сигналы механического сдвига почвы и объектов поиска. Таким образом реализуется двухканальная схема работы миноискателя, включающая получение информации о тангенсе угла потерь $\operatorname{tg} \varphi=\frac{\sigma}{\omega \varepsilon}$, даваемом радиоволновым каналом, и параметр механического сдвига $\Delta x$, где $\varepsilon$ - диэлектрическая проницаемость почвы; $\sigma$ - ее электропроводность; $\omega$ - рабочая частота радиоволнового канала. Передатчик

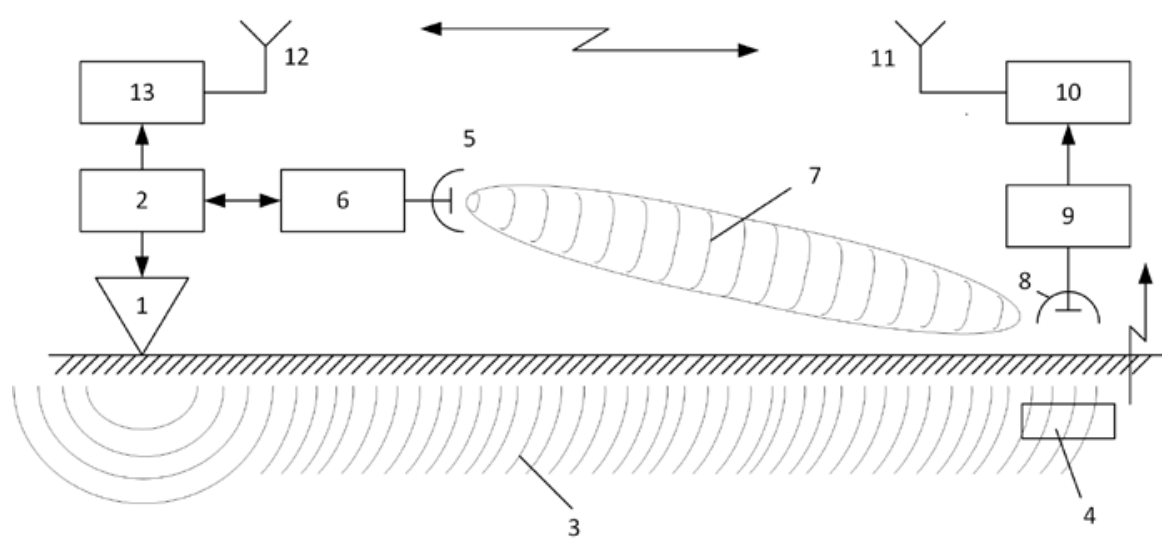

Рис. 3. Схема возбуждения и приема механических колебаний корпуса и мины

Fig. 3. Scheme of excitation and reception of mechanical vibrations of the mines 


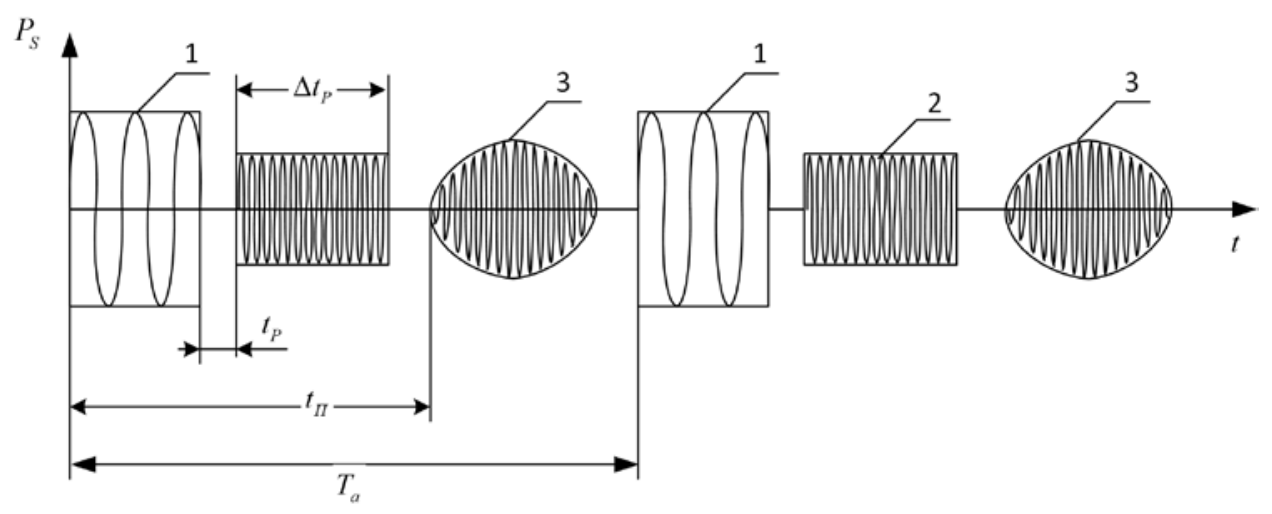

Рис. 4. Временная диаграмма излучения и приема

Fig. 4. Timing diagram of radiation and reception

Таблица 1

Table 1

\begin{tabular}{|c|c|c|c|c|}
\hline $\begin{array}{c}\text { Среда } \\
\text { распространения }\end{array}$ & Песок & Сухая почва & Супесь & Глина \\
\hline Скорость $V_{a}, \mathrm{M} / \mathrm{c}$ & $100-500$ & 200 & 300 & $800-2000$ \\
\hline
\end{tabular}

классического радиоволнового миноискателя располагается совместно с приемником на поисковом элементе БР.

В табл. 1 представлена зависимость скорости поперечных $V_{a}$ сейсмических волн для различных видов почв [5].

Как видим, наименьшую скорость распространения сейсмических волн имеет песок $-V_{a}$ до $100 \mathrm{M} / \mathrm{c}$.

При заданной максимальной дальности действия сейсмического вибратора, $r_{a}=100$ м, время запаздывания сейсмической волны составит порядка $t_{\Pi}=1$ с. Откуда период повторения сейсмического сигнала с учетом времени работы радиоволнового канала должен быть $T_{a}>1$ с. Поскольку на расстоянии $r_{a}=100$ м время запаздывания ЭМ сигнала незначительно, то радиопередатчик - 6 может излучать с запаздыванием во времени относительно сейсмического импульса на время $t_{I T}=0,9 \mathrm{c}$.

Далее оценим необходимую чувствительность приемника РЛС, если будем использовать фазовый метод демодуляции отраженного от поверхности почвы сигнала. В соответствии с [2] амплитуда механического смещения корпуса мины под действием сейсмического воздействия силой удара $P_{a}(\mathrm{H})$ можно оценить как

$$
\Delta x \approx \frac{P_{a}}{4 \pi R_{a}^{2}} \cdot \frac{R_{a}}{r_{a}} S \frac{Q}{m} \tau^{2},
$$

где $R_{a}$ - радиус излучающей плиты сейсмоисточника; $S\left(\boldsymbol{M}^{2}\right)$ - площадь боковой поверхности мины; $m(\kappa 2)$ - ее масса; $\tau(c)$ - длительность полупериода возбуждающего сейсмического 
удара; $P_{a}(H)$ - сила удара; $r_{a}(M)$ - расстояние от сейсмоисточника до мины; $Q$ - добротность корпуса.

Как видно из (1), амплитуда механического смещения корпуса объекта $\Delta x$ в первую очередь зависит от отношения $S / m$, т.е. от площади его поверхности к массе. Этот параметр специфичен для мин разного типа, поэтому может быть заранее внесен в базовую память системы расспознавания.

При фазовом методе демодуляции сигнала от мины смещение фазы отраженного радиосигнала составит:

$$
\Delta \varphi=\omega \frac{\Delta h}{v_{\ni}},
$$

где $\omega$ - рабочая частота ЭМ сигнала; $\Delta h$ - смещение поверхности почвы; $v_{\ni}=\frac{c}{\sqrt{\varepsilon}}-$ скорость ЭМ волны в почве; $\varepsilon$ - диэлектрическая проницаемость почвы.

По данным экспериментальных исследований, вертикальный сдвиг объекта примерно соответствует горизонтальному $\Delta h \approx \Delta x$.

С учетом глубины слоя скин-эффекта ЭМ волны в почве при ожидаемом заглублении мины $h \approx 0,3$ м рабочая длина ЭМ волны должна быть этого же порядка. Поэтому целесообразной рабочей частотой, разрешенной к применению для этих целей регламентом радиосвязи, может быть $f_{э} \approx 2,5$ ГГи с учетом потерь в грунте.

Пусть $\Delta h=10^{-6} \mathcal{M} ; \varepsilon=9$; тогда из (2) получаем:

$$
\Delta \varphi=2 \pi f_{\ni} \frac{\Delta h}{v_{\ni}}=2 \pi f_{\ni} \frac{\Delta h \sqrt{\varepsilon}}{c} \approx 0,01 \text { град. }
$$

Откуда следует, что чувствительность приемника РЛС по амплитуде должна соответствовать полупериоду несущей частоты, т.е. оцениваться по отношению к сдвигу фаз $180^{\circ}$ :

$$
\delta_{E}=\frac{\Delta E}{E}=\frac{\Delta \varphi}{180^{0}} \approx 1 \cdot 10^{-4}
$$

При требовании в отношении сигнал/шум порядка 10 дБ общая необходимая чувствительность приемника составит $\delta_{E}=1 \cdot 10^{-5}$.

Поскольку при минимальной фазовой манипуляции радиосигнала ее индекс соответствует амплитудной модуляции, то в итоге требуемое отношение сигнал/шум по амплитуде должно быть не менее $q_{E}=\frac{1}{\delta_{E}}=1 \cdot 10^{5}=50$ дБ.

Далее дадим оценку требуемой мощности сейсмического удара.

Пусть $\Delta x=10^{-6} \mathrm{M} ; R_{a}=0.1 \mathrm{M}^{2} ; r_{a}=100 \mathrm{M} ; \mathrm{m}=10$ кг; $\tau=5 \cdot 10^{-3}$ с. В этом случае

$$
P_{a}=1.7 \cdot 10^{4} \mathrm{H} .
$$

На фото (рис. 5) показан экспериментальный сейсмический вибратор с силой удара $500 \mathrm{H}$.

Потенциальным резервом для увеличения смещения $\Delta x$, кроме длительности полупериода удара $\tau^{2}$, является также в целом длительность виброимпульса $\tau_{B}$. 


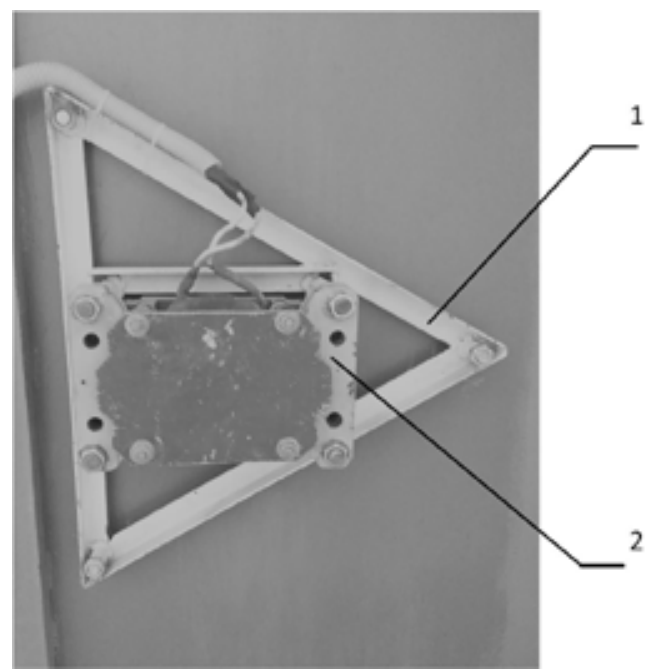

Рис. 5. Экспериментальный сейсмический вибратор: 1 - опорная рама; 2 - электромагнит

Fig. 5. Experimental Seismic Vibrator: 1 - support frame; 2 - electromagnet

При средней частоте механического резонанса мины $F_{M} \approx 20$ Ги [2] и длительности виброимпульсного воздействия $\tau_{B} \approx 250$ мс производится 5 ударов вибратора, что равносильно увеличению силы удара в 5 раз. Это повышает отношение сигнал/шум на величину в 20 дБ и составит $q_{E}=80$ дБ. Длительность вибрационного воздействия определяется скоростью движения поискового аппарата.

Оценим требуемую напряженность первичного ЭМ поля через собственный шум приемника. Будем исходить из необходимой полосы частот приемника $\Delta F_{M}$ для всех возможных собственных частот механических колебаний мин разного типа - от противопехотных до противотанковых.

В этом случае мощность теплового шума на входе приемника

$$
P_{U}=\kappa T_{K}^{0} \Delta F_{M} q_{W},
$$

где $\kappa=1.38 \cdot 10^{-23}$ - постоянная Больцмана; $T_{K}^{0}$ - температура входных цепей по Кельвину; $q_{\amalg}=50$ дБ - коэффициент шума; $\Delta F_{M}$ - полоса пропускания приемника.

Сигнал первичного поля приемника с учетом ослабления вторичного поля мины в 50 дБ

$$
P_{\Pi P}=P_{U} \cdot 10^{5} .
$$

В итоге имеем при $\Delta F_{M}=1000$ Гц $q_{\amalg}=50$ дБ

$$
P_{\text {IP }}=\kappa T_{K}^{0} \Delta F_{M} q_{U I} \cdot 10^{5}=4,14 \cdot 10^{-6} \mathrm{BT} .
$$

Требуемую мощность передатчика $P_{\Pi}$ РЛС получим через известное уравнение:

$$
P_{\Pi}=\frac{4 \pi P_{\Pi P} r^{2}}{Q_{\Pi} S_{\Pi P}} .
$$


Здесь $Q_{\Pi}=4 \pi \frac{S_{\Pi}}{\lambda^{2}} \eta-$ коэффициент направленного действия антенны передатчика; $S_{\Pi P} \approx \frac{\lambda^{2}}{4}-$ эффективная площадь элемента приемной антенны поискового БР в виде рупора.

При длине волны, соответствующей рабочей частоте РЛС 2,5 ГГи $\lambda=0,12 \mathrm{м}$, эффективной площади передающей антенны РЛС $S_{\Pi}=0.1 \mathrm{~m}^{2}$ и КПД антенны $\eta=0,5$, на максимальном расстоянии до объекта $r=100$ м, требуемая мощность передатчика $P_{\text {II }}$ РЛС не превышает 1 мВт.

\section{Аппаратный комплекс, система и технология работы}

Количество периферийных роботов-носителей может составить 5-6 на борт с соответствующим числом операторов. Управление производится по видеоканалу с размещением видеокамер как на самом роботе-носителе, так и на машине - операторе. Представляет интерес конструкция периферийных управляемых роботов-носителей датчиков обнаружения мин. Это могут быть высокопроходимые самодвижущиеся машины массой 10-50 кг для обработки открытых площадей. Поскольку масса полезного груза миноискателей небольшая 1-2 кг, возможна установка на БПЛА квадрокоптеров. Процесс сканирования местности обеспечивает оператор.

Принцип работы электромеханического вибратора показан на рис. 6. Он содержит ударную плиту - 1, ударник с электромеханическим приводом - 2 и возбудитель вибратора - 3, синхронизируемый от бортового синтезатора частот -4 .

Конструктивно вибратор размещается в металлическом кожухе в виде саней, подвешивается на корме базовой машины и приводится в контакт с грунтом гидравлическим приводом.

Поисковый элемент, собственно двухканальный миноискатель, устанавливается на подвеске робота-носителя БР, управляемого по видеоканалу оператором базовой машины. Предварительная обработка информации и перевод в цифровую форму производится в самих элементах миноискателей и передается оператору базовой машины по радиоканалу либо по проводной линии.

Технология работы заключается в том, что задачу по обследованию площади с базовой машины выполняют периферийные управляемые устройства БР - носители миноискателей.

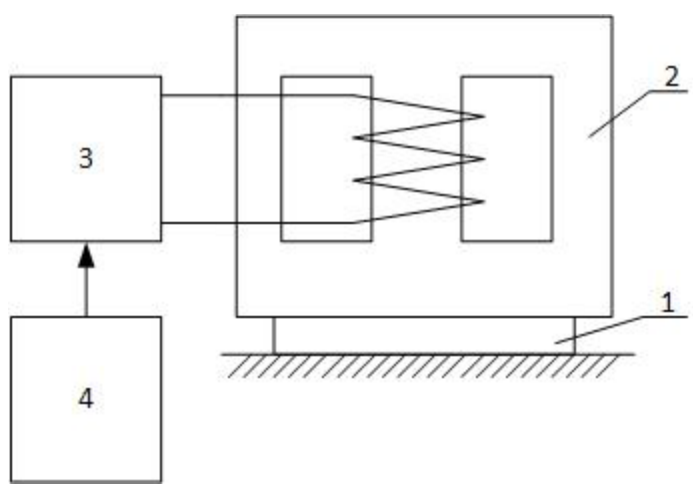

Рис. 6

Fig. 6 
Базовая машина останавливается, начинает работать вибратор, а БР обследуют заминированную площадь на расстоянии 10-100 м вдоль трассы поиска с шириной полосы 10 м, охватываемой БР.

При обнаружении мины, БР отводится оператором от этого места, возможно навигатором или какой-либо меткой отмечается местоположение объекта, а потом решается задача уничтожения. Таким образом реализуется задача безопасного разминирования с увеличением производительности поиска.

\section{Заключение}

1. Описанный в статье аппаратный комплекс может быть использован в первую очередь для гуманитарного разминирования больших площадей.

2. Двухканальный принцип построения поискового элемента в сочетании радиоволнового искателя и параметрического, с привлечением дополнительного признака распознавания мины - механического смещения корпуса под действием виброударов, позволяет снижать число ложных тревог, увеличив вероятность верного обнаружения цели и тем самым ускорив процесс поиска.

3. Физические основы работы комплекса не противоречат основным законам электро- и сейсмодинамики и могут быть реализованы в достаточно короткое время с учетом актуальных проблем.

Работа выполнена при финансовой поддержке РФФИ в рамках научного проекта № 18-07-00180 A.

\section{Список литературы}

[1] Щербаков Г.Н., Анцелевич М.А. Новые методы обнаружения скрытых объектов. М.: ООО «Эльф ИПР», 2011. 503 с. [Shcherbakov G.N, Ancelevich M.A. New methods for detecting hidden objects. M.: OOO «Alf IPR», 2011. 503 P. (in Russian)]

[2] Шайдуров Г.Я., Кудинов Д.С. Дистанционный метод поиска минно-взрывных заграждений и мин в любых корпусах на основе взаимодействия электромагнитных и акустических волн. Специальная техника, 2011, 4, 39-43 [Shaydurov G.Y, Kudinov D.S. Remote method of mineexplosive obstacles and mines search in any hulls on the basis of interaction of electromagnetic and acoustic waves, Special equipment, 2011, 4, 39-43 (in Russian)]

[3] Шайдуров Г.Я. Пат. № 2390801 РФ. Способ поиска объектов искусственного происхождения в земле и устройство для его осуществления. Зарегистрировано в Гос. реестре изобретений РФ 27 мая 2010 г. [Shaydurov G.Y. Pat. No. 2390801 RU. The method of searching for objects of artificial origin in the earth and the device for its implementation. Registered in the Register of inventions of the Russian Federation 27 may 2010 (in Russian)]

[4] Robert W. Haupt., Kenneth D. Rolt. Standoff Acoustic Laser Technique to Locate Buried Land Mines, Lincoln laboratory journal, 2005, 1 (15), 3-23

[5] Бондарев В.И. Сейсморазведка. Екатеринбург: УГГА, 2007. 690 с. [Bondarev V.I. Seismic exploration. Ekaterinburg, UGGA, 2007, 690 p. (in Russian)] 